\title{
Using Estimation and Projection Package and Spectrum for Jamaica's national HIV estimates and targets
}

\author{
Jacqueline Duncan, Sharlene Beckford Jarrett, Kevin Harvey
}

Ministry of Health, Kingston, Jamaica

\section{Correspondence to} Dr Jacqueline Duncan, Ministry of Health, 2-4 King Street, Kingston, Jamaica; jjjduncan@yahoo.com

Accepted 27 September 2010

\begin{abstract}
Background Adequate coverage of target populations ensures that desired outcomes, such as increased survival of people living with HIV, are achieved. However, estimates of coverage and impact of HIV programmes using available data are limited by the complex natural history of HIV, underreporting of cases and inadequate information systems.
\end{abstract}

Methods Jamaica's national HIV estimates were generated using the 2009 version of the UNAIDS estimation and projection package (EPP) and Spectrum. National data used included sentinel surveillance data on antenatal clinic attendees (1986-2005 and 2007), distribution of antiretroviral regimes for prevention of mother-to-child transmission, distribution of antiretroviral therapy (ART) among adults and ART distribution in subpopulations (eg, men who have sex with men (MSM) and sex workers). Surveys of MSM (2007), sex worker (2001, 2005, 2008), STI clinic attendees (1990-2002) and inmates (2006) also provided inputs.

Results In 2009, Jamaica's HIV prevalence was estimated at 1.7\% (range 1.1-2.5) and 31000 (range $20000-43000$ ) adults (>15 years) were living with HIV. The number of adults in need of treatment was 15000 (range 11000-19000) and approximately 2100 new infections occurred in 2009. The EPP/Spectrum estimates were generally consistent with locally available data. However, the number of persons with advanced HIV targeted by the national treatment programme was significantly lower than Spectrum's estimated target population.

Conclusion EPP/Spectrum can provide important data for national HIV programme planning. Improved monitoring and evaluation systems will provide quality data and result in more robust estimates.

The HIV epidemic in Jamaica has features of both a generalised and concentrated epidemic. The adult HIV prevalence was estimated at $1.6 \%$ in 2007 and 27000 person were living with HIV. ${ }^{1}$ However, higher HIV prevalence has been found in the most at-risk populations such as sex workers ( $9 \%$ ), men who have sex with men (MSM; 32\%), crack/cocaine users $(5 \%)$, inmates $(3.3 \%)$ and sexually transmitted infection (STI) clinic attendees (3.6\%). ${ }^{2}{ }^{3}$ Several behavioural factors such as early sexual debut, high levels of multiple partnership, transactional sex and limited condom use increase HIV transmission in Jamaica. These factors coupled with gender disparities, poverty and homophobia provide fertile ground for the growth of the HIV epidemic and reinforce the need for a multisectoral response. ${ }^{2}$
Jamaica's national HIV/STI programme (NHP) has identified four priority areas in order to halt and reverse the spread of HIV: prevention; treatment care and support; enabling environment and empowerment and governance. ${ }^{4}$ Key indicators and targets are used to monitor progress towards universal access. However, the quantity of services required for achieving access to all those who need services and, hence target-setting, are hindered by insufficient data on populations in need of services.

Currently, the HIV surveillance system and national surveys provide information on the HIV epidemic in Jamaica and identify populations in need of services such as treatment. The surveillance system is a name-based confidential reporting system that began in 1982. Between 1982 and 2008, 23972 persons living with HIV, including 13445 persons with AIDS and 7394 AIDS deaths, were reported to the Ministry of Health. ${ }^{3}$ The number of persons reported with HIV per year doubled between 1996 (971 cases) and 2008 (1868 cases). This is due to new HIV infections as well as increased access to testing, improved surveillance and increased awareness about HIV/AIDS among healthcare workers and the general population. Public access to antiretroviral therapy (ART) in 2004 coupled with improved laboratory capacity and support services resulted in a $40 \%$ decrease in AIDS deaths between 2004 and 2008. ${ }^{3}$

Despite improvements in HIV surveillance, many gaps persist and hamper accurate generation of estimates. For example, inclusion in the surveillance system relies on persons being tested and diagnosed, but nearly half of people living with HIV are unaware of their status. ${ }^{2}$ A previous survey of Jamaican physicians revealed as much as $30-40 \%$ underreporting of people living with HIV accessing care. ${ }^{5}$ In addition, case report forms are often incomplete, limiting the understanding of the contribution of the most at-risk populations in Jamaica's HIV epidemic. For example, although HIV transmission is primarily through heterosexual transmission, the sexual practice of $43 \%$ of men reported with HIV is unknown. Similarly, the risk history is unknown for at least $20 \%$ of persons reported with AIDS. $^{3}$ In addition, limited data are available on the progression of HIV and the impact of interventions, including ART availability, in Jamaica. These limitations can have a negative impact on programme planning and accurate target-setting, and further limit the ability of the NHP to measure efforts to halt the transmission of HIV reliably.

Surveillance of high-risk groups such as sex workers and MSM have been challenging, partly 
due to stigma and discrimination, which makes accessing these populations difficult. Despite these challenges, the NHP completed second-generation behavioural surveillance activities with these groups between 2001 and 2008, along with smaller studies.

In 2009, the NHP sought to strengthen its understanding of Jamaica's HIV epidemic and populations in need of services by participating in the UNAIDS estimates process using the estimation and projection package (EPP) and Spectrum.

\section{METHODS}

The NHP began data gathering exercises in 2009 in preparation for the estimates process using UNAIDS EPP and Spectrum software. The software is based on recommendations of the UNAIDS reference group on estimates, modelling and projections and incorporates latest research findings on the epidemiology of HIV. The objectives of EPP for concentrated epidemics are to build models of national epidemics using key subpopulations, which may include the most at-risk populations or geographically diverse subepidemics. The output of EPP served as input to Spectrum for assessing incidence trends, ART and prevention of mother-to-child transmission (PMTCT) needs. The EPP software and the Spectrum model have been described in detail elsewhere. ${ }^{6} 7$

The main data required for EPP were characteristics of subpopulations (size, demography and duration in the subpopulation), distribution of first and second-line ART among the general population and subpopulations, antenatal clinic sentinel surveillance data and prevalence data from surveys of the most at-risk populations. The EPP output provided data on HIV incidence with the aggregate national adult incidence curve as the key input for Spectrum. Other data required for estimates in Spectrum included the distribution of antiretroviral regimes among HIV-infected pregnant women for PMTCT, distribution of feeding practices among HIV-positive mothers, the proportion of persons with advanced HIV on ART per year and the distribution of cotrimoxazole treatment and ART among children.

\section{Data on HIV prevalence}

The data on HIV prevalence came from the national HIV sentinel surveillance, second-generation behavioural surveillance studies and special studies (table 1). Sentinel surveillance of antenatal clinic and STI clinic attendees were conducted annually between 1986 and 2007 (excluding 2006). Sentinel surveillance was conducted at urban and rural public antenatal clinic sites between April and September of each year.

Second-generation behavioural surveillance of the most at-risk populations have generally been smaller and less frequent. The

Table 1 Summary of surveillance activities among subpopulations in Jamaica

\begin{tabular}{ll}
\hline Subpopulation & Data source, (years of data collection) \\
\hline MSM & Second-generation behavioural surveillance (2007) \\
Sex workers & $\begin{array}{l}\text { Second-generation behavioural surveillance }(2005,2008) . \\
\text { Special study }(1994-5,2001)\end{array}$ \\
Client of sex workers & STI clinic sentinel surveillance (1990-2002) \\
Crack/cocaine users & Drug rehabilitation programme monitoring data (2004) \\
Prison inmates & Special study (2006) \\
Male remaining & Antenatal sentinel surveillance (annual collection \\
population & $1986-2005,2007)$ \\
Female remaining & Antenatal sentinel surveillance (annual collection \\
population & $1986-2005,2007)$ \\
\hline
\end{tabular}

MSM, men who have sex with men. second-generation surveillance of sex workers conducted in 2001 included 660 women from establishments and street venues. The 2005 survey, however, was composed of 450 women who represented largely street-based sex workers and included HIV testing. The 2008 survey comprised 277 women from establishments and street venues. In all three surveys, women were recruited in the capital city as well as rural and tourist sites.

The first national second-generation surveillance of MSM was undertaken in 2007. This survey included 201 men who were recruited from four parishes, representing urban, rural and tourist resort areas. Prevalence data for prison inmates and drug using populations were gathered from special studies conducted in the prison setting and drug rehabilitation facilities.

In order to fit a curve to the MSM and sex workers data, results from smaller studies were also included in EPP, on the recommendation of experts from UNAIDS.

\section{Characteristics of subpopulations}

The subpopulations identified for Jamaica's estimates process were sex workers, clients of sex workers, MSM, inmates, crack/ cocaine users, the remaining male population and the remaining female population. Injecting drug users were not included as surveillance data shows that less than $1 \%$ of people living with HIV in Jamaica report injecting drug use as a risk factor. ${ }^{3}$ Estimation of subpopulation sizes was based on studies in the Caribbean region, national survey data, national census data and a review of monitoring data from outreach workers. ${ }^{8-10}$

To estimate the number of MSM and sex workers, UNAIDS guidelines on the prevalence of MSM and same-sex activity was applied to the adult male and female populations, respectively, as a starting point. Next, the estimates were adjusted based on local mapping data from outreach activities. For example, the UNAIDS estimate of MSM activity was reduced from 5\% to $3 \%$ of the adult male population for Jamaica.

Estimates of the number of crack/cocaine users were based on the National Household Survey (2001) and the National School Survey (2006). The estimate of the number of prison inmates was restricted to subpopulations thought to be at higher risk of exposure to HIV due to engaging in same-sex activity while incarcerated, drug use, or multiple partnerships.

To estimate the number of clients of sex workers, data on the number of clients reported in surveys was considered along with monitoring data from outreach workers at sex sites.

Estimates of the duration of time that persons were engaged in high-risk behaviours in the groups that had population movement were made based on feedback from HIV/AIDS experts from UNAIDS during the estimates workshop, and when available key questions in surveys such as the age distribution of persons surveyed in the risk group and median age for MSM and sex workers. Table 2 summarises subpopulation characteristics used in the estimates process.

\section{Data on PMTCT programme}

The distribution of ART among HIV-infected pregnant women was determined from PMTCT programme monitoring reports. A combination of paper-based and electronic reporting systems collect information on all HIV-infected pregnant women delivering in the public sector and their antiretroviral regimes. This system is likely to capture most HIV-infected women in the public sector as more than $95 \%$ of women attending public antenatal clinics receive HIV testing, and rapid HIV testing is available on labour wards for women who present at a late stage. Private sector coverage of HIV-infected pregnant women is likely 
Table 2 Summary of characteristics of subpopulations in Jamaica

\begin{tabular}{lcllll}
\hline & $\begin{array}{l}\text { Population } \\
\text { estimate } \\
>15 \text { years } \\
\text { old }\end{array}$ & $\begin{array}{l}\text { Average } \\
\text { time spent } \\
\text { in group } \\
\text { (years) }\end{array}$ & $\begin{array}{l}\text { Prevalence } \\
\text { (\%) }\end{array}$ & $\begin{array}{l}\text { \% on } \\
\text { first } \\
\text { line } \\
\text { ART }\end{array}$ & $\begin{array}{l}\text { \% } \\
\text { Male }\end{array}$ \\
\hline MSM $^{*}$ & 27999 & 10 & 31.8 & 21.0 & 1.00 \\
Female sex workers & 12000 & 10 & 9.0 & 5.0 & 0.00 \\
Male clients of sex workers & 46000 & 5 & 3.6 & 9.4 & 1.00 \\
Crack/cocaine users & 4500 & 10 & 5.0 & 0.3 & 0.5 \\
Prison inmates & 5000 & 10 & 3.3 & 1.3 & 1.00 \\
Male remaining population & 802945 & NA & 1.3 & 18 & 1.00 \\
Female remaining & 985217 & NA & 1.3 & 45 & 0.00 \\
Papulation & & & & &
\end{tabular}

population

*A later recommendation to the estimates process is that turn-over for men who have sex with men (MSM) should be from high-risk to low-risk MSM instead of turning over to the male remaining population.

ART, antiretroviral therapy.

to be similar to public sector coverage based on national surveys, which confirm that more than $90 \%$ of pregnant women in Jamaica receive HIV testing (public and private sector). ${ }^{6} 7 \mathrm{We}$ estimated that less than $5 \%$ of HIV-infected pregnant women received ART before 2002 as ART access was costly and limited to the private sector before 2002. In 2008, $83 \%$ of HIV-infected pregnant women received ART for PMTCT and $98 \%$ of HIV-exposed infants received ART in 2008.

The feeding practices of HIV-positive mothers are not captured routinely by the monitoring and evaluation system. However, data on breastfeeding were obtained from a UNICEF nutrition study conducted in 2008, which used a convenience sample of 189 HIV-positive mothers. ${ }^{11}$ Ninety-eight per cent of mothers reported exclusive formula feeding.

Data on the number of infants receiving PCR was obtained from the national public health laboratory, which was the only laboratory conducting PCR in Jamaica at that time. Data on co-trimoxazole prophylaxis in children was not available as it is not routinely captured by the monitoring and evaluation system.

\section{Data on ART programme}

Public access to ART in Jamaica began in September 2004. Before 2004, very little data were available on ART use, which was accessible in the private sector only. We assumed that less than $10 \%$ of persons in need of treatment were accessing ART before 2004 and second-line treatment was non-existent. The distribution of first and second-line ART after 2004 was obtained from pharmacy records and an electronic ART register. The pharmacy data were used to estimate the number of persons on treatment per year. The electronic treatment registers were available for nine of 18 treatment sites island-wide. The distribution of ART regimes across these nine representative sites was extrapolated to the population on treatment annually. At a minimum, the electronic registers capture data on patient demographics, CD4 cell counts and ART prescribed at each clinic visit.

Data on the distribution of ART among subpopulations was not available. Clinical experience and expert opinion on access to care by the subpopulations were used to estimate the distribution of ART among subpopulations.

\section{Assumptions of the EPP and Spectrum software}

Increased availability of data from cohort studies and national surveys has improved the assumptions of the software. Some key assumptions of the software used in the Jamaica estimates process include:

1. A median time from infection to death of 10.5 years for men and 11.5 years for women. The time from infection to eligibility and from eligibility to death was based on CD4 cell counts of less than 200 and less than 350 cells/ $\mu 1$.

2. Average annual survival on ART is approximately $86 \%$ in the first year and $90 \%$ in subsequent years.

3. As ART coverage increases, the median CD4 cell count at ART initiation will rise and first year survival will also rise, nearing $95 \%$ as ART coverage nears $100 \%$.

4. First year survival depends on the baseline CD4 cell count.

5. Transmission from mother to child may be during pregnancy, delivery and through breastfeeding. Intrapartum transmission depended on the efficacy of various PMTCT regimens.

6. The probability of transmission through breastfeeding depends on the type and duration of breastfeeding. Mixed feeding has the highest risk and mothers on ART to treat HIV have the lowest risk.

7. The ratio of fertility among HIV-infected women to fertility among HIV-uninfected women is above 1 for younger age groups (15-19 years) but below 1 for older HIV-infected women.

All data were reviewed and endorsed by the Jamaica Monitoring and Evaluation Reference Group.

\section{RESULTS}

Jamaica's adult HIV prevalence was estimated to be $1.7 \%$ $(1.1-2.5)$ in 2009 and 31000 persons (20000-43000) were living with HIV (table 3). This is higher than prevalence estimates from sentinel surveillance data, which showed that $1.16 \%$ of public sector antenatal clinic attendees were HIV positive. Both Spectrum outputs and sentinel surveillance data suggest there has been no significant change in HIV prevalence over the past decade (figure 1). The Spectrum output indicates that the male:female ratio for people living with HIV is approximately 2:1 compared with 1.3:1 based on HIV surveillance data.

The number of persons in need of treatment was estimated at 15000 when a CD 4 cell count of 350 cells/ $\mu$ l was used as the set point for treatment initiation based on local guidelines. Based on programme monitoring, 6895 persons (6459 adults and 436 children) with advanced HIV (49\% of persons with advanced HIV) were started on treatment at the end of December 2009.

\section{DISCUSSION}

Universal access to treatment services is an international priority in the HIV response, but accurate planning of services to achieve this goal is hindered by insufficient data on the HIV epidemic. Various statistical models have been developed to determine the extent of the HIV epidemic. ${ }^{12}$ Improved assumptions and increased availability of data have improved the robustness of the estimates generated. ${ }^{12} 13$ Jamaica's HIV response had limited data on HIV prevalence, the number of children living with HIV and persons with advanced HIV. The previous prevalence

Table 3 Comparison of HIV estimates from Spectrum with HIV surveillance data

\begin{tabular}{lll}
\hline & $\begin{array}{l}\text { 2009 Estimates } \\
\text { based on HIV } \\
\text { surveillance data (CI) }\end{array}$ & $\begin{array}{l}\text { 2009 Estimates } \\
\text { Spectrum output (CI) }\end{array}$ \\
\hline HIV prevalence, adults & $1.40 \%$ & $1.7(1.1$ to 2.5$)$ \\
HIV population, adults & 27000 & $31000(20000$ to 43000$)$ \\
HIV population, women & 11600 & $10000(6700$ to 14 000) \\
No of new HIV infections & NA & $2100(<1000$ to 4200) \\
No of AIDS deaths, adults & 600 & $1200(<500$ to 2100) \\
No of persons in need of ART & 8500 & $15000(11000$ to 19 000)
\end{tabular}

ART, antiretroviral therapy. 


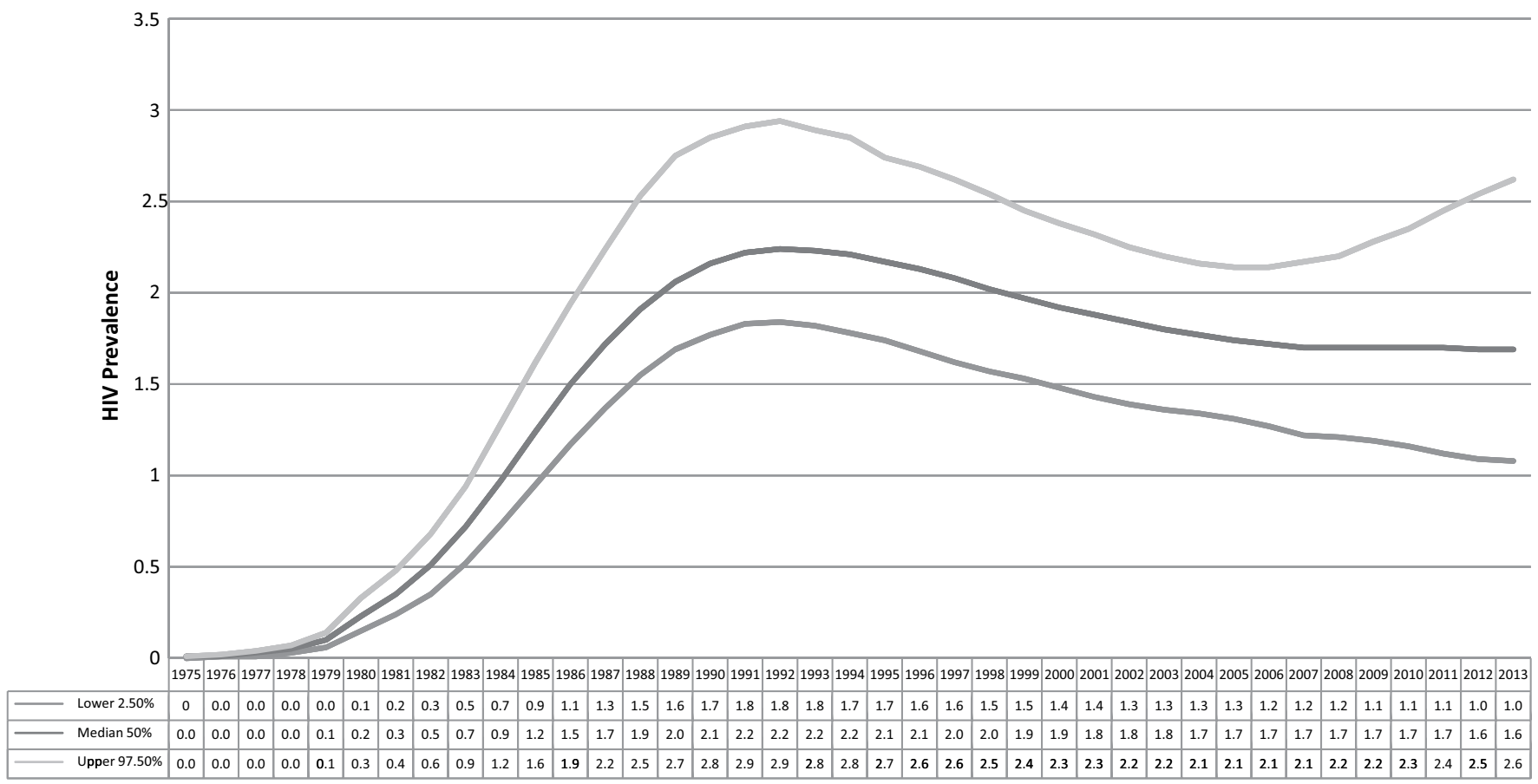

Figure 1 Spectrum projection of adult HIV prevalence in Jamaica.

estimates in Jamaica relied on sentinel surveillance data from antenatal clinic populations only, which may distort our understanding of the epidemic. The EPP and Spectrum software strengthened the estimate process by considering subpopulations such as MSM, sex workers as well as the impact of interventions such as ART, PMTCT and population dynamics. In addition, it provided data on areas that were otherwise unavailable such as HIV incidence.

In particular, the estimates process assisted with target setting for the measurement of progress towards universal access to services. For example, based on surveillance data, Jamaica's treatment programme estimated that 8500 persons had advanced HIV and would be in need of treatment in 2009. However, the Spectrum output suggests that 15000 persons (range 11000-19000) are in need of treatment, placing the national estimates well outside the software's lower estimate (table 4). This discrepancy may be due to underestimation of persons with advanced HIV using surveillance data. Many persons with advanced HIV are undiagnosed and are not captured by the surveillance system. This served as an impetus to review strategies to identify persons in need of treatment and resulted in a revision of targets for the HIV treatment programme from 500 to 1000 new persons on treatment per year.

Jamaica's estimates process also identified possible gaps in the HIV response. For example, the estimated number of AIDS deaths was significantly higher than the number of reported AIDS deaths in 2008. This may reflect underdiagnosis rather than underreporting as death registers are routinely reviewed as part of active surveillance. Other strategies for improving case detection must be considered, including post mortem diagnoses.

The generation of these estimates also highlights the need to review how epidemics are classified. First, HIV prevalence in the general population in Jamaica is consistently above $1 \%$ over the past decade. ${ }^{14}$ By definition, Jamaica's HIV epidemic has features of both a generalised and concentrated epidemic. However, we opted to use projections for a concentrated epidemic as the software does not allow countries to select both a generalised and concentrated epidemic option. The implications on the estimates generated is not clear but sensitivity analyses indicated that the HIV prevalence estimates were generally less when the generalised epidemic option was used. The estimates process highlights the need to review definitions for describing the HIV epidemic in countries such as Jamaica without ignoring important sub-epidemics, particularly among MSM.

Second, many assumptions of the model were based on studies in Africa, Europe and Latin America. Data from the Caribbean was limited or non-existent and assumptions may not reflect the Jamaican situation. For example, unlike other countries where heterosexual transmission is the primary mode of transmission, reported AIDS cases among men continue to exceed AIDS cases among women in Jamaica. ${ }^{1}$ This was contrary to the assumptions about the male:female ratio in the software. However, countries are allowed to use their own data if available and modify assumptions. As data collection systems in the Caribbean and in Jamaica improve and provide countryspecific data on survival, treatment efficacy and HIV progression, the Spectrum estimates will become increasingly reliable and informative.

Local data were unavailable or limited in many instances, for example, the most at-risk population sizes and duration in risk group. Variations in these inputs revealed significant changes in estimates generated and male:female ratios. This emphasised the

Table 4 Spectrum estimate of persons in need of treatment based on CD4 cell criteria

\begin{tabular}{lll}
\hline & \multicolumn{2}{l}{$\begin{array}{l}\text { Spectrum estimate of number in need of ART } \\
\text { median, (range) CD4 cell count }<350 \text { cells/ } \mu \text { I }\end{array}$} \\
\cline { 2 - 3 } & $\mathbf{2 0 0 8}$ & $\mathbf{2 0 0 9}$ \\
\hline Adults $(15$ years +$)$ & $15000(11000-18000)$ & $15000(11000-19000)$ \\
Mothers in need of PMTCT & $<500(<500-<1000)$ & $<500(<500-<1000)$ \\
Children $0-14$ years & $<500(<500-<1000)$ & $<500(<500-<1000)$ \\
No of AIDS deaths, adults & $1600(<1000-2400)$ & $1200(<500-2100)$ \\
\hline
\end{tabular}

ART, antiretroviral therapy; PMTCT, prevention of mother-to-child transmission. 


\section{Key messages}

- Jamaica's estimates process provided epidemiological data that assisted with strategic planning.

- Some of the assumptions of the estimates process may not be applicable to countries in the Caribbean, such as Jamaica, and may distort projections.

- More research and improvement in monitoring and evaluation systems are necessary to strengthen the estimates process.

importance of gaining consensus on the inputs and outputs for the process as well as understanding the limitations of the estimates generated.

Finally, the estimates process considered the impact of ART treatment and PMTCT coverage in projecting new infections and deaths. However, the impact of prevention programmes and the expansion of HIV testing on projections are not included. These interventions are central in Jamaica's HIV response. The impact of such interventions on new HIV infections is less clear, but these factors should be considered in HIV incidence and prevalence estimates. In addition, assessment of time trends in prevalence estimates is not possible due to methodological changes and variations of assumptions with each model. ${ }^{12}$

Despite these limitations the estimates process provided data that increased the Jamaica NHP's understanding of the HIV epidemic and populations in need of service. Future estimates will be more accurate as more data become available and countries improve their monitoring and evaluation systems.

Competing interests None declared.
Contributors JD was the primary author who conceptualised and guided the questions, analysis and discussion. SBJ analysed data and contributed to writing and revision of the article. $\mathrm{KH}$ contributed to the conceptualisation and analysis of data.

Provenance and peer review Not commissioned; externally peer reviewed.

\section{REFERENCES}

1. UNAIDS/WHO. AIDS epidemic update - December 2008. Geneva: UNAIDS/WHO, 2008.

2. Figueroa JP, Duncan J, Byfield L, et al. A comprehensive response to the HIV/AIDS epidemic in Jamaica, a review of the past 20 years. West Indian Med $\mathrm{J}$ 2008;57:562

3. Ministry of Health, Jamaica. Jamaica HIV epi update January to December, 2008. Jamaica: Ministry of Health, 2009.

4. National HIV/STI Programme, Jamaica. National strategic plan 2007-2012. Jamaica: Ministry of Health, 2007.

5. Figueroa JP, Brathwaite AR. Is under-reporting of AIDS a problem in Jamaica? West Indian Med J 1995;44:51-4.

6. Stover J, Johnson P, Hallett T, et al. The Spectrum projection package: improvements in estimating incidence by age and sex, mother-to-child transmission, HIV progression in children and double orphans. Sex Transm Infect 2010;86(Suppl 2): ii16-21.

7. Brown T, Bao L, Raftery AE, et al. Modelling HIV epidemics in the antiretroviral era: the UNAIDS estimation and projection package 2009. Sex Transm Infect 2010;86 (Suppl 2):ii3-10.

8. Caceres C, Konda K, Pecheny $M$, et al. Estimating the number of men who have sex with men in low and middle income countries. Sex Transm Infect 2006;82(Suppl 3): iii3-9.

9. FHI. HIV/AIDS Behavioural Surveillance Survey Jamaica, 1999-2000. http://www. fhi.org/en/CountryProfiles/Jamaica/jamaicatools.htm (accessed 1 0ct 2007).

10. De La Haye W, Powell K, Pinnock S, et al. Profile and pattern of substance abuse in clients diagnosed with HIV in a substance abuse treatment unit in a general hospital in Jamaica. West Indian Med J 2004;53(Suppl):35.

11. Southern Regional Health Authority. Annual PMTCT nutritionist report. Mandeville, Jamaica: Southern Regional Health Authority, 2008.

12. Brookmeyer R. Measuring the HIV/AIDS epidemic: approaches and challenges. Epidemiol Rev 2010;32:26-37.

13. Mahy M, Warner-Smith M, Stanecki KA, et al. Measuring the impact of the global response to the AIDS epidemic: challenges and future directions. J Acquir Immune Defic Syndr 2009;52(Suppl 2):S152-9.

14. National HIV/STI Programme. HIV Senitnel Surveillance Serosurvey Report 2007 Jamaica: Ministry of Health, 2007. 